\title{
COMPACT AND EXTREMALLY DISCONNECTED SPACES
}

\author{
BHAMINI M. P. NAYAR
}

Received 22 August 2002

\author{
To Prof. James E. Joseph on His 65th Birthday
}

\begin{abstract}
Viglino defined a Hausdorff topological space to be $C$-compact if each closed subset of the space is an $H$-set in the sense of Veličko. In this paper, we study the class of Hausdorff spaces characterized by the property that each closed subset is an $S$-set in the sense of Dickman and Krystock. Such spaces are called $C$-s-compact. Recently, the notion of strongly subclosed relation, introduced by Joseph, has been utilized to characterize $C$-compact spaces as those with the property that each function from the space to a Hausdorff space with a strongly subclosed inverse is closed. Here, it is shown that $C$-s-compact spaces are characterized by the property that each function from the space to a Hausdorff space with a strongly sub-semiclosed inverse is a closed function. It is established that this class of spaces is the same as the class of Hausdorff, compact, and extremally disconnected spaces. The class of $C$-s-compact spaces is properly contained in the class of $C$-compact spaces as well as in the class of $S$-closed spaces of Thompson. In general, a compact space need not be $C$-s-compact. The product of two $C$-s-compact spaces need not be $C$-s-compact.
\end{abstract}

2000 Mathematics Subject Classification: 54D25, 54C99, 54D30, 54G05.

1. Introduction. It is well known that every continuous function from a compact space to a Hausdorff space is a closed function. In 1969, Viglino [15] introduced the family of $C$-compact spaces, showing that every continuous function from a $C$-compact space to a Hausdorff space is a closed function, and that this class of spaces properly contains the class of compact spaces. A space $X$ is $C$-compact if for every closed subset $A$ of $X$ and for every cover of $A$ by open subsets of $X$, there is a finite subfamily whose closures in $X$ will cover $A$. In 1970, Goss and Viglino [4] provided a Hausdorff space which is not $C$-compact but which has the property that every continuous function from the space to a Hausdorff space is a closed function. In 1969, Dickman and Zame [3] introduced functionally compact spaces, a class of spaces which is characterized by the property that every continuous function from any one of its members to a Hausdorff space is a closed function. Since then, a number of researchers (see [1, 4, 5, 8, 10, 12] to mention a few) have studied these classes of spaces and a great deal of effort has been given to the problem of identifying a class of functions such that a space $X$ is $C$ compact if and only if each function in this class from the space $X$ to a Hausdorff space is closed. Very often, the result of such an effort was the introduction of new classes of spaces. Throughout this paper, all spaces are assumed to be Hausdorff. Definitions of the concepts used are given in Section 2.

In [10], the notion of strongly subclosed relation, introduced by Joseph [6], was used to give the following solution to this problem. 
THEOREM 1.1. A space $X$ is C-compact if and only if all functions on $X$ with strongly subclosed inverses are closed functions.

Motivated by this discovery, in [8], Joseph et al. studied the following two classes of spaces, each of which contains the class of countably compact spaces as well as the class of $C$-compact spaces.

(1) Each continuous function from the space maps closed subsets onto sequentially closed subsets.

(2) Each function on the space with a strongly subclosed inverse maps closed subsets onto sequentially closed subsets.

Spaces satisfying (1) are called sequentially functionally compact and those satisfying (2) are called sequentially $C$-compact. Spaces satisfying (2) also satisfy (1). The present paper is also a study in this direction. In 1976, Thompson [13] introduced the concept of an $S$-closed space while studying compact extremally disconnected Hausdorff spaces in an $H$-closed model using the concept of a semiopen subset of Levine [9]. A space $X$ is $S$-closed if every cover of $X$ with semiopen sets has a finite subfamily whose closures cover $X$. This concept generated considerable interest among researchers in this field. Parallel to the concept of an $H$-set, the concept of an $S$-set was introduced by Dickman and Krystock [2]. A subset $A$ of $X$ is an $S$-set if every cover of $A$ by semiopen subsets of $X$ has a finite subfamily whose closures in $X$ cover $A$. In this paper, a space $X$ is $C$ $s$-compact if every cover of every closed subset $A$ of $X$, by semiopen subsets of $X$, has a finite subfamily whose closures in $X$ cover $A$. In other words, a $C$-s-compact space is a space for which every closed subset is an $S$-set. Here, using the concept of functions with strongly sub-semiclosed graphs introduced by Joseph and Kwack [7], the class of $C$-s-compact spaces is characterized by each of the following:

(a) each function from $X$ to a Hausdorff space $Y$ with a strongly sub-semiclosed inverse is a closed function;

(b) each function from $X$ to a Hausdorff space $Y$ with a strongly sub-semiclosed inverse is a semiclosed function.

In [1], Clay and Joseph proved that a Hausdorff space $Y$ is $C$-compact if and only if every strongly subclosed relation from a Hausdorff space $X$ to $Y$ is upper semicontinuous, denoted as u.s.c. Recall that a relation $R$ from $X$ to $Y$ is a subset of $X \times Y$, where the domain of $R$ is $X$. A relation $R$ from $X$ to $Y$ will be denoted by $R \subseteq X \times Y$. In Theorem 3.10, we show that a space $Y$ is $C$-s-compact if and only if for every space $X$, every strongly sub-semiclosed relation from $X$ to $Y$ is u.s.c.

In [3], Dickman and Zame defined a space to be functionally compact if each open filterbase $\Omega$ on the space satisfying ad $\Omega=\bigcap_{\Omega} F$ converges to $\bigcap_{\Omega} F$. Theorem 3.2 establishes that a space $X$ is $C$-s-compact if and only if each open filterbase $\Omega$ is an open-set base for $\operatorname{sad} \Omega$.

In 1966, Veličko [14] introduced the concept of an $H$-set. A subset $A$ of a Hausdorff space $X$ is an $H$-set if every cover of $A$, by open subsets of $X$, contains a finite subfamily whose closures in $X$ cover $A$. Since the introduction of this concept, it has been widely used in the study of $H$-closed-type properties. In fact, $C$-compact spaces are those spaces for which every closed subset is an $H$-set.

Among other characterizations, the following theorem is proved. 
THEOREM 1.2. A space $X$ is $C$-s-compact if and only if every nonempty proper closed subset of $X$ is an $S$-set.

In view of the above result and [2, Proposition 4.1], in the class of Hausdorff spaces, the property of being $C$-s-compact is equivalent to the property of being compact and extremally disconnected. It is known that every compact and extremally disconnected space is homeomorphic to the Stone space of some complete Boolean algebra. Thus a $C$-s-compact space is homeomorphic to the Stone space of some complete Boolean algebra. In Section 3, characterizations of and related results for $C$-s-compact spaces are obtained using covers, filterbases, relations satisfying certain graph conditions, and $S$-sets. In Section 4, examples are given to distinguish the class of $C$-s-compact spaces from the classes of compact spaces, $S$-closed spaces, and $C$-compact spaces. It is clear that a $C$-s-compact space is $C$-compact as well as $S$-closed. Examples are given to show that an $S$-closed space need not be $C$-s-compact. Also, a $C$-compact space might fail to be $C$-s-compact. An example of a compact space which is not $C$-s-compact is provided. Products of two $C$-s-compact spaces need not be $C$-s-compact. The basic definitions and notations used in the paper are found in the following section.

2. Preliminaries. Let $A$ be a subset of a space $X$. Closure and interior of $A$ are denoted by $\mathrm{cl} A$ and int $A$, respectively. The family of all open subsets of $X$ containing $A$ will be denoted by $\Sigma(A)(\Sigma(x)$, if $A=\{x\})$. A set $A \subseteq X$ is said to be semiopen [9] if there is an open set $U \subseteq X$ such that $U \subseteq A \subseteq \operatorname{cl} U$. The family of all semiopen subsets of $X$ containing $A$ is denoted by $\mathrm{SO}(A)(\mathrm{SO}(x)$, if $A=\{x\})$. A point $x$ is a semi-interior point of $A$ if there is a $B \in \mathrm{SO}(x)$ contained in $A$. The semi-interior of $A$ is denoted by $\operatorname{sint} A$, and $A$ is semiopen if and only if $A=\operatorname{sint} A$. A point $x$ is in the semiclosure of $A$ if $V \cap A \neq \varnothing$ for every $V \in \mathrm{SO}(x)$. The semiclosure of $A$ is denoted by $\operatorname{scl} A$, and $A$ is semiclosed if $A=\operatorname{scl} A$. The $\theta$-closure [14] (resp., $\theta$-semiclosure [7]) of $A$, denoted as $\operatorname{cl}_{\theta} A$, (resp., $\operatorname{scl}_{\theta} A$ ) is $\bigcap_{\Sigma(A)} \operatorname{cl} V$ (resp., $\{x \in X$ such that $\operatorname{cl} V \cap A \neq \varnothing$ for every $V \in \operatorname{SO}(x)\}$ ). A set $A \subseteq X$ is regularly open (resp., regularly closed) if $A=\operatorname{int}(\operatorname{cl} A)$ (resp., $A=\operatorname{cl}(\operatorname{int} A)$ ) or equivalently interior of a closed set (resp., closure of an open set). Since the family of regularly closed subsets of a space $X$ is the same as the family of closures of semiopen subsets of $X$, then $x \in \operatorname{scl}_{\theta} F$ if and only if for every regularly closed set $V \subseteq X$ containing $x, V \cap F \neq \varnothing$. The $\theta$-adherence [14] (resp., $s$-adherence, $s$ - $\theta$-adherence) of a filterbase $\Omega$, denoted as $\operatorname{ad}_{\theta} \Omega$ (resp., $\operatorname{sad} \Omega, \operatorname{sad}_{\theta} \Omega$ ) on $X$, is $\bigcap_{\Omega} \mathrm{cl}_{\theta} F$ (resp., $\bigcap_{\Omega} \operatorname{scl} F$, $\bigcap_{\Omega} \operatorname{scl}_{\theta} F$ ). The $s$ - $\theta$-adherent point and $s$-adherent point of a filterbase are called $s$ accumulation point and $s_{*}$-accumulation point in [2], respectively. It is clear that for a set $A, \operatorname{scl}_{\theta} A \subseteq \mathrm{cl}_{\theta} A$, and for a filterbase $\Omega$, every $s$-adherent point is an adherent point and an $s$ - $\theta$-adherent point is a $\theta$-adherent point. A filterbase $\Omega$ is $s$-adherent convergent if every neighborhood of the $s$-adherent set of $\Omega$ contains a member of $\Omega$. Let $X$ and $Y$ be topological spaces. A relation $R \subseteq X \times Y$ is strongly subclosed (has a strongly subclosed graph [6]) (resp., strongly sub-semiclosed) if $\operatorname{ad}_{\theta} R(\Omega) \subseteq R(x)\left(\operatorname{resp} ., \operatorname{sad}_{\theta} R(\Omega) \subseteq R(x)\right.$ ) for each $x \in X$ and filterbase $\Omega$ on $X-\{x\}$ with $\Omega \rightarrow x$. In [7], a function with a strongly sub-semiclosed graph is called a function with $\theta$-semi-subclosed graph. A function $g \subseteq X \times Y$ has a strongly subclosed inverse (resp., strongly sub-semiclosed inverse) if $g^{-1} \subseteq g(X) \times X$ is strongly subclosed (resp., strongly sub-semiclosed). Clearly, 
a strongly subclosed relation $R \subseteq X \times Y$ is strongly sub-semiclosed. It is known that continuous, and indeed $\theta$-continuous, functions have strongly subclosed inverses. A relation $R \subseteq X \times Y$ is u.s.c. [1], if for each $x \in X$ and $W \in \Sigma(R(x))$ in $Y$, there is a $V \in \Sigma(x)$ in $X$ such that $R(V) \subseteq W$. A function $f: X \rightarrow Y$ is said to be semiclosed [11] if $f(A)$ is semiclosed for each closed $A \subseteq X$. A space $X$ is extremally disconnected if the closure of every open set is open. Therefore, in an extremally disconnected space, every regularly closed set is open. Throughout the paper, unless otherwise stated, all operations are taken with respect to the original space.

\section{3. $C$-s-compact spaces}

Definition 3.1. A space $(X, T)$ is $C$-s-compact if, whenever $A$ is a closed subset of $X$ and $\Omega$ is a $T$-semiopen cover of $A$, there is a finite subfamily $\Lambda$ of $\Omega$ such that $A \subseteq \bigcup_{\Lambda} \operatorname{cl} U$, closures taken in the original topology.

Equivalently, $X$ is $C$-s-compact if every closed subset is $S$-closed, relative to $X$. Obviously, a $C$-s-compact space is $C$-compact as well as $S$-closed. But as will be seen in Section 4 , an $S$-closed space as well as a $C$-compact space might fail to be $C$-s-compact.

THEOREM 3.2. The following are equivalent for a space $(X, T)$ :

(a) $X$ is $C$-s-compact;

(b) for each closed set $A \subseteq X$ and each filterbase $\Omega$ on $A, A \cap \operatorname{sad}_{\theta} \Omega \neq \varnothing$;

(c) for each closed set $A \subseteq X$ and each open filterbase $\Omega$ on $A, A \cap \operatorname{sad} \Omega \neq \varnothing$;

(d) each open filterbase on $X$ is s-adherent convergent;

(e) each open filterbase $\Omega$ is an open-set base for $\operatorname{sad} \Omega$;

(f) if $A$ is a closed subset of $X$ and $\Gamma$ is a family of semiclosed subsets of $X$ such that $\left(\cap_{\Gamma} G\right) \cap A=\varnothing$, then there is a finite $\Lambda \subseteq \Gamma$ such that $\left(\bigcap_{\Lambda} \operatorname{int} G\right) \cap A=\varnothing$;

(g) if $A$ is a closed subset of $X$ and $\Omega$ is an open filterbase on $X$ whose members have nonempty intersection with $A$, then there is an s-adherent point of $\Omega$ in $A$;

(h) given a closed subset $A$ of $X$, a T-semiopen cover $\Gamma$ of $X-A$, and an open neighborhood $V$ of $A$, there is a finite $\Lambda \subseteq \Gamma$ such that $X=V \cup\left(\cup_{\Lambda} \operatorname{cl} U\right)$.

Proof. The proof will follow the sequence $(\mathrm{a}) \Rightarrow(\mathrm{b}) \Rightarrow(\mathrm{c}) \Rightarrow(\mathrm{d}) \Rightarrow(\mathrm{e}) \Rightarrow(\mathrm{f}) \Rightarrow(\mathrm{g}) \Rightarrow(\mathrm{a}),(\mathrm{g}) \Rightarrow$ $(\mathrm{h}) \Rightarrow(\mathrm{d})$.

(a) $\Rightarrow$ (b). Let $X$ be $C$-s-compact and let $A$ be a closed subset of $X$. Let $\Omega$ be a filterbase on $A$ and suppose that $A \cap \operatorname{sad}_{\theta} \Omega=\varnothing$. Then for each $a \in A$, there is an $F_{a} \in \Omega$ and $a \notin \operatorname{scl}_{\theta} F_{a}$. Hence, for each $a \in A$, there is a $U_{a} \in \operatorname{SO}(a)$ such that $\operatorname{cl} U_{a} \cap F_{a}=\varnothing$. The family $U=\left\{U_{a}: a \in A\right\}$ is a semiopen cover of $A$. Choose a finite $B \subseteq A$ such that $A \subseteq \bigcup_{B} \mathrm{cl} U_{a}$. Now, choose $F \in \Omega$ such that $F \subseteq \bigcap_{B} F_{a}$. A contradiction of the fact that $\operatorname{cl} U_{a} \cap F_{a}=\varnothing$ for every $a \in B$ is reached since $\operatorname{cl} U_{a} \cap F \neq \varnothing$ for some $a \in B$ and $F \subseteq F_{a}$ for every $a \in B$.

(b) $\Rightarrow$ (c). Let $A$ be a closed subset of $X$ and let $\Omega$ be an open filterbase on $A$. Suppose that $A \cap \operatorname{sad}(\Omega)=\varnothing$. Then for each $a \in A$, there is an $F_{a} \in \Omega$ and a $U_{a} \in \operatorname{SO}(a)$ such that $U_{a} \cap F_{a}=\varnothing$. Since $F_{a}$ is open, it follows that for each $a \in A, \operatorname{cl} U_{a} \cap F_{a}=\varnothing$. This contradicts (b).

(c) $\Rightarrow$ (d). Let $\Omega$ be an open filterbase on $X$ and let $A$ be the $s$-adherent set of $\Omega$. Then $A$ is nonempty. Let $U$ be an open neighborhood of $A$. Then $\{X-\operatorname{scl} F: F \in \Omega\}$ is a semiopen 
cover of the closed set $X-U$. Hence, there is a finite $\Lambda \subseteq \Omega$ such that $X-U \subseteq \bigcup_{\Lambda} \operatorname{cl}(X-$ $\operatorname{scl} F) \subseteq \bigcup_{\Lambda}(X-F)=X-\bigcap_{\Lambda} F$. Hence, $\bigcap_{\Lambda} F \subseteq U$. Thus, $\Omega$ is $s$-adherent convergent.

$(\mathrm{d}) \Rightarrow(\mathrm{e})$. The proof follows immediately.

(e) $\Rightarrow$ (f). Let $A$ be a closed subset of $X$ and let $\Gamma$ be a family of semiclosed subsets of $X$ such that $\left(\bigcap_{\Gamma} U\right) \cap A=\varnothing$. Suppose that for every finite $\Lambda \subseteq \Gamma,\left(\bigcap_{\Lambda} \operatorname{int} U\right) \cap A \neq \varnothing$. Then $\left\{\left(\bigcap_{\Lambda} \operatorname{int} U\right) \cap A: \Lambda\right.$ finite, $\left.\Lambda \subseteq \Gamma\right\}$ is an open filterbase on $A$. Hence, $\mathscr{V}=\left\{\left(\bigcap_{\Lambda} \operatorname{int} U\right)\right.$ : $\Lambda$ finite, $\Lambda \subseteq \Gamma\}$ is an open filterbase on $X$. Then

$$
\operatorname{sad} \mathscr{V}=B=\bigcap_{\mathscr{V}} \operatorname{scl} V=\bigcap_{\text {finite } \Lambda \subseteq \Gamma} \operatorname{scl}\left(\bigcap_{\Lambda} \operatorname{int} U\right) \subseteq \bigcap_{\text {finite } \Lambda \subseteq \Gamma}\left(\bigcap_{\Lambda} \operatorname{scl}(\operatorname{int} U)\right) .
$$

Since each $U$ is semiclosed and $\left(\bigcap_{\Gamma} U\right) \cap A=\varnothing, X-A$ is an open set containing $B$. But no $V \in \mathscr{V}$ is contained in $X-A$. This contradicts (e).

(f) $\Rightarrow(\mathrm{g})$. Let $A$ be a closed subset of $X$ and let $\Omega$ be an open filterbase on $X$ whose members have nonempty intersection with $A$. Suppose that $(\operatorname{sad} \Omega) \cap A=\varnothing$. Then $\{\operatorname{scl} F: F \in \Omega\}$ is a family of semiclosed sets such that $\left(\bigcap_{\Omega} \operatorname{scl} F\right) \cap A=\varnothing$. Hence, in view of (f), choose a finite $\Lambda \subseteq \Omega$ such that $\left(\bigcap_{\Lambda} \operatorname{int}(\operatorname{scl} F)\right) \cap A=\varnothing$. Since each $F$ is open, this means that $\left(\bigcap_{\Lambda} F\right) \cap A=\varnothing$. This contradicts the assumption since $\Omega$ is a filterbase on $X$ whose members have nonempty intersection with $A$.

$(\mathrm{g}) \Rightarrow(\mathrm{h})$. Let $A$ be a closed subset of $X$ and let $V$ be an open neighborhood of $A$. Let $\Gamma$ be a semiopen cover of $X-A$. Then $\Gamma$ is a semiopen cover of the closed set $X-V$. Suppose that there is no finite $\Lambda \subseteq \Gamma$ satisfying $X-V \subseteq \bigcup_{\Lambda} \operatorname{cl} U$. Then $\mathscr{V}=\{W: W=$ $(X-V)-\bigcup_{\Lambda \subseteq \Gamma} \operatorname{cl} U, \Lambda$ finite $\}$ is an open filterbase on the closed set $X-V$. Therefore, in view of $(\mathrm{g}),\left(\operatorname{sad}^{\mathscr{V}}\right) \cap(X-V) \neq \varnothing$. Hence, $\left(\bigcap_{\gamma} \operatorname{scl} W\right) \cap(X-V) \neq \varnothing$. Then $\left(\bigcap_{\Gamma} \operatorname{scl}(X-\right.$ $\operatorname{cl} U)) \cap(X-V) \neq \varnothing$. Hence, $(X-V) \cap\left(X-\bigcup_{\Gamma} \operatorname{sint}(\operatorname{cl} U)\right) \neq \varnothing$. Since each $U$ is semiopen, $U \subseteq \operatorname{sint}(\operatorname{cl} U)$. Therefore, $\left(X-\bigcup_{\Gamma} U\right) \cap(X-V) \neq \varnothing$, a contradiction to the fact that $\Gamma$ is a cover of $X-V$. Therefore, $X=\left(\bigcup_{\Lambda} \operatorname{cl} U\right) \cup V$ for some finite $\Lambda \subseteq \Gamma$.

$(\mathrm{g}) \Rightarrow(\mathrm{a})$. Let $A$ be a closed subset of $(X, T)$ and let $U$ be a $T$-semiopen cover of $A$. Assume that there is no finite $\mathscr{S} \subseteq \mathcal{U}$ satisfying $A \subseteq \bigcup_{\mathscr{S}} \operatorname{cl} U$. Consider $\mathscr{V}=\{V: V=$ $\bigcap_{\mathscr{C} \subseteq \mathscr{U}}(X-\operatorname{cl} U), \mathscr{S}$ finite $\}$. Then $\mathscr{V}$ is an open filterbase on $X$ and $V \cap A \neq \varnothing$ for each $V \in \mathscr{V}$. Then proceeding as in the proof of $(\mathrm{g}) \Rightarrow(\mathrm{h})$, a contradiction to the fact that $U$ is a cover of $A$ is reached.

(h) $\Rightarrow$ (d). Let $\Omega$ be an open filterbase on $X$ and let $A=\operatorname{sad} \Omega$. Let $U$ be an open neighborhood of $A$. Then $\mathscr{V}=\{X-\operatorname{scl} F: F \in \Omega\}$ is a semiopen cover of $X-A$ and hence of $X-U$. Also, considering $U$ as a neighborhood of $X-(X-U)$, in view of $(h)$, choose a finite $\Gamma \subseteq \Omega$ such that $X=U \cup\left(\bigcup_{\Gamma} \operatorname{cl}(X-\operatorname{scl} F)\right)$. Thus $X=U \cup\left(\bigcup_{\Gamma}(X-\operatorname{int}(\operatorname{scl} F))\right)$. Hence, $X \subseteq U \cup\left(X-\bigcap_{\Gamma} F\right)$. Choose an $F \in \Omega$ such that $X=U \cup(X-F)$. Then $F \subseteq U$ and $\Omega$ is $s$-adherent convergent.

As mentioned earlier, a $C$-compact space and a sequentially $C$-compact space [8] are characterized using a family of $H$-sets of the space. Below, a characterization of $C$-s-compact spaces is given in terms of a family of proper subsets which are $S$-sets.

THEOREM 3.3. A space $X$ is a $C$-s-compact space if and only if every nonempty proper closed subset of $X$ is an $S$-set. 
Proof. Assume that every nonempty proper closed subset of $X$ is an $S$-set. It will be enough to prove that $X$ is $S$-closed. Let $U$ be a semiopen cover of $X$. Assume that each $U \in \mathcal{U}$ is proper, semiopen, and nonempty. Choose a $U \in \mathcal{U}$ and an open set $F$ in $X$ such that $F \subseteq U \subseteq \operatorname{cl} F$. Now, $X-F$ is a nonempty proper closed subset of $X$ and hence is an $S$-set. Also, $U$ is a semiopen cover of $X-F$. Choose a finite $\mathscr{Y} \subseteq U$ such that $X-F \subseteq \bigcup_{\mathscr{S}} \mathrm{cl} U$. Then $X=\left(\bigcup_{\mathscr{S}} \mathrm{cl} U\right) \cup U$ and hence, $X$ is $S$-closed.

It is obvious that if $X$ is $C$-s-compact, every proper closed subset is an $S$-set.

COROLlaRY 3.4. A space $X$ is C-s-compact if and only if every closed subset is an $S$-set.

COROLLARY 3.5. A space $X$ is $C$-s-compact if and only if $X$ is compact and extremally disconnected.

Proof. The proof follows easily from [2, Corollary 2.4 and Proposition 4.1] which establish that every closed subset of a space $X$ is an $S$-set if and only if $X$ is compact and extremally disconnected.

COROLLARY 3.6. The following are equivalent for a space $X$ :

(a) $X$ is $C$-s-compact;

(b) let $A$ be a nonempty proper closed subset of $X$, then for every filterbase $\Omega$ on $A$, $A \cap \operatorname{sad}_{\theta} \Omega \neq \varnothing ;$

(c) let $A$ be a nonempty proper closed subset of $X$, then for every open filterbase $\Omega$ on $A, A \cap \operatorname{sad} \Omega \neq \varnothing$.

Proof. The proof follows from Theorem 3.3 and [2, Proposition 2.2].

In [10], a class of functions was identified so that a space $X$ is $C$-compact if and only if a function in this class on the space $X$ is a closed function. Here, a class of functions is identified, which characterizes $C$-s-compact spaces similarly.

THEOREM 3.7. The following are equivalent for a space $X$ :

(a) $X$ is $C$-s-compact;

(b) each function $g$ from $X$ to a space $Y$ with a strongly sub-semiclosed inverse is a closed function;

(c) each function $g$ from $X$ to a space $Y$ with a strongly sub-semiclosed inverse is a semiclosed function.

Proof. (a) $\Rightarrow($ b). Let $X$ be $C$-s-compact, let $A$ be a closed subset of $X$, and let $g$ : $X \rightarrow Y$ have a strongly sub-semiclosed inverse. Let $y$ be a limit point of $g(A)$. Then $\Omega=\left\{g^{-1}(W-\{y\}) \cap A: W \in \Sigma(y)\right\}$ is a filterbase on $A$. In view of Theorem 3.2(b), $A \cap \operatorname{sad}_{\theta} \Omega \neq \varnothing$. But $A \cap \operatorname{sad}_{\theta} \Omega \subseteq A \cap g^{-1}(y)$ since $g$ has a strongly sub-semiclosed inverse. Thus, $A \cap g^{-1}(y) \neq \varnothing, y \in g(A)$, and $g(A)$ is closed.

(b) $\Rightarrow$ (c). The proof is obvious.

(c) $\Rightarrow$ (a). Suppose that $X$ is not $C$-s-compact. Appealing to Corollary 3.6, choose a proper closed subset $A$ of $X$ and a filterbase $\Omega$ on $A$ with $A \cap \operatorname{sad}_{\theta} \Omega=\varnothing$. Let $v \in X-A$. Let $Y$ be the set $X$ with the topology $\{V \subseteq Y: v \notin V$, or $F \subseteq V$ for some $F \in \Omega\}$. The space $Y$ is easily shown to be Hausdorff. Each $B \in \mathrm{SO}(v)$ in $Y$ satisfies $B \cap F \neq \varnothing$ for 
each $F \in \Omega$, and consequently $v \in \operatorname{scl} A-A$ in $Y$. Suppose otherwise and let $B \in \operatorname{SO}(v)$ in $Y$. Choose an open set $U \subseteq B \subseteq \operatorname{cl} U$. If $v \in U$, then $U$ contains an $F \in \Omega$. Suppose that $v \notin U$ and $B \cap F=\varnothing$ for some $F \in \Omega$. For such an $F,(F \cup\{v\}) \cap U=\varnothing$. So, $v \notin \operatorname{cl} U$, a contradiction. Hence, each $B \in \mathrm{SO}(v)$ in $Y$ satisfies $B \cap F \neq \varnothing$ for each $F \in \Omega$. Let $g: X \rightarrow Y$ be defined as $g(x)=x$ for $x \in A$ and $g(x)=v$ for $x \in X-A$. Then $g^{-1} \subseteq \mathcal{g}(X) \times X$ has a strongly sub-semiclosed graph for, if $\Lambda$ is a filterbase on $g(X)-\{y\}$ with $\Lambda \rightarrow y$ in $Y$, then $y=v$ and $\operatorname{sad}_{\theta} g^{-1}(\Lambda) \subseteq \operatorname{sad}_{\theta} \Omega \subseteq g^{-1}(v)$. So, $A$ is closed in $X, g(A)=A$, and $v \in \operatorname{scl} A-A$ in $Y$. Thus, $g$ is not semiclosed.

REMARK 3.8. In view of the facts that a function with a strongly subclosed graph has a strongly sub-semiclosed graph and every closed function is semiclosed, the following corollary is an improvement of the sufficiency of the main result of [10] which states that a space $X$ is $C$-compact if and only if each function on $X$ with a strongly subclosed inverse is a closed function.

COROLlary 3.9. A space $X$ is $C$-compact if every function on $X$ with a strongly subsemiclosed inverse is semiclosed.

Proof. The proof is immediate since every $C$-s-compact space is $C$-compact.

THEOREM 3.10. A space $Y$ is $C$-s-compact if and only if for every space $X$, every strongly sub-semiclosed relation from $X$ to $Y$ is u.s.c.

Proof. Suppose that $Y$ is $C$-s-compact and $R$ is a strongly sub-semiclosed relation from a space $X$ to $Y$. Let $x \in X$ and $W \in \Sigma(R(x))$ in $Y$. If $\{x\}$ is open, then $\{x\} \in \Sigma(x)$ and $R(\{x\}) \subseteq W$. Suppose that $\{x\}$ is not open. Let $V \in \Sigma(x)$ in $X$. Then $\Omega=\{V-\{x\}$ : $V \in \Sigma(x)\}$ is a filterbase on $X-\{x\}$ and $\Omega \rightarrow x$. Suppose that $R(V-\{x\}) \cap(Y-W) \neq \varnothing$ is satisfied for each $V \in \Sigma(x)$. Then $\Gamma=\{R(V-\{x\}) \cap(Y-W): V \in \Sigma(x)\}$ is a filterbase on the closed subset $Y-W$ of the $C$-s-compact space $Y$. Hence, in view of Theorem 3.2, $\left(\operatorname{sad}_{\theta} \Gamma\right) \cap(Y-W) \neq \varnothing$. Since $R$ is a strongly sub-semiclosed relation, $\operatorname{sad}_{\theta} R(V-\{x\}) \subseteq$ $R(x)$ and hence, $\operatorname{sad}_{\theta} R(V-\{x\}) \cap(Y-W) \subseteq R(x) \cap(Y-W)$. This is a contradiction since $R(x) \subseteq W$. Therefore, there is a $V \in \Sigma(x)$ such that $R(V-\{x\}) \subseteq W$ and $R(x) \subseteq W$. Hence, there is a $V \in \Sigma(x)$ such that $R(V) \subseteq W$. Therefore, $R$ is a u.s.c. relation.

Conversely, suppose that $Y$ is not $C$-s-compact. Relying on Corollary 3.6(b), choose a nonempty proper closed subset $A$ of $Y$ and a filterbase $\Omega$ on $A$ with $A \cap \operatorname{sad}_{\theta} \Omega=\varnothing$. Let $v \in Y-A$. Let $X$ be the set $Y$ with the topology $\{V \subseteq X: v \notin V$, or $F \subseteq V$ for some $F \in$ $\Omega\}$. The space $X$ is readily seen to be Hausdorff. Define a relation $R \subseteq X \times Y$ as follows: $(x, x) \in R$ if $x \neq v ;(v, x) \in R$ if $x \in X-A$. The relation $R$ is strongly sub-semiclosed for, if $\Gamma$ is a filterbase on $X-\{x\}$ with $\Gamma \rightarrow x$ in $X$, then $x=v$. Also, $\operatorname{sad}_{\theta} R(\Gamma)=\operatorname{sad}_{\theta} \Omega \subseteq$ $Y-A=R(v)$. But $R$ is not u.s.c. since $Y-A \in \Sigma(R(v))$ in $Y$, and there is no $V \in \Sigma(v)$ in $X$ such that $R(V) \subseteq Y-A$.

4. Examples. In this section, examples are provided to distinguish the class of $C$ - $s$ compact spaces from the classes of $C$-compact spaces, compact spaces, and $S$-closed spaces. Since there exist compact spaces which are not $S$-closed and $S$-closed spaces which are not compact, there exist compact spaces which are not $C$-S-compact and $S$-closed spaces which are not $C$-s-compact. However, such examples are pointed out for the sake of completeness. Also, an example is given to show that the product of 
two $C$-s-compact spaces need not be $C$-s-compact. From the definitions of $C$-s-compact spaces and $C$-compact spaces and from the fact that every open set is semiopen, it is clear that a $C$-s-compact space is $C$-compact. The following example (see [15, Example $2]$ ) is an example of a Hausdorf $C$-compact space which is not compact and hence not $C$-s-compact.

EXAMPLE 4.1. Let $\mathbb{N}$ represent the set of positive integers. Let $Y$ denote the subset of the plane consisting of all points of the form $(1 / n, 1 / m)$ and points of the form $(1 / n, 0)$ for $n$ and $m$ in $\mathbb{N}$. Let $X=Y \cup\{\infty\}$. Topologize $X$ as follows: let each point $(1 / n, 1 / m)$ be open. Partition $\mathbb{N}$ into infinitely many infinite equivalence classes $\left\{\mathbb{N}_{i}\right\}_{i=1}^{\infty}$. A neighborhood $U(1 / i, 0)$ of $(1 / i, 0)$ is of the form $\{(1 / i, 0)\} \cup\{(1 / i, 1 / m): m \geq k\} \cup$ $\left\{(1 / n, 1 / m): m \in \mathbb{N}_{i}, n \geq k\right\}$ for some $k \in \mathbb{N}$. A neighborhood system for the point $\infty$ consists of all sets of the form $X-T$, where

$$
T=\left\{\left(\frac{1}{n}, 0\right): n \in \mathbb{N}\right\} \cup\left(\bigcup_{i=1}^{k}\left(\left\{\left(\frac{1}{i}, \frac{1}{m}\right): m \in \mathbb{N}\right\} \cup\left\{\left(\frac{1}{n}, \frac{1}{m}\right): m \in \mathbb{N}_{i}, n \in \mathbb{N}\right\}\right)\right)
$$

for some $k \in \mathbb{N}$. The space $X$ with this topology is Hausdorff and is not compact. Hence, it is not $C$-s-compact. It can also be verified that for the closed set $G=\{(i / n, 0)$ : $n \in \mathbb{N}\}$ and the semiopen cover $\mathcal{U}=\left\{U_{n}: U_{n}=\{(1 / n, 1 / m): m \geq k\right.$ for some $k \in$ $\mathbb{N}\} \cup\{(1 / n, 0)\}\}$ of $G$, there is no finite $\mathscr{S} \subseteq \mathcal{U}$ such that $G \subseteq \bigcup_{\mathscr{S}} \operatorname{cl} U$.

The following example illustrates that, in general, a compact space need not be $C$-scompact.

EXAMPLE 4.2. Let $X=\bigcup_{i=0}^{\infty} X_{i}$, where $X_{0}=\{(0,1 / n): n \in \mathbb{N}\} \cup\{(0,0)\}$ and $X_{i}=$ $\{(1 / i, 1 / n): n \in \mathbb{N}\} \cup\{(1 / i, 0)\}$ for $i \geq 1$. For $i \geq 1$, each point of $X_{i}$, except $(1 / i, 0)$, is open. Basic open subsets

$$
U\left(\frac{1}{i}, 0\right)=\left\{\left(\frac{1}{i}, 0\right)\right\} \cup\left\{\left(\frac{1}{i}, \frac{1}{n}\right): n>j \text { for some } j \in \mathbb{N}\right\} .
$$

Basic open subsets $U(0,1 / i)=\{(0,1 / i)\} \cup\{(1 / n, 1 / i)$ for all but finitely many $n \in \mathbb{N}\}$. A basic open set containing $(0,0)$ contains all the points on all but finitely many $X_{i}$, $i \in \mathbb{N}$. Then $X$ is compact since an open set containing $(0,0)$ contains all the points on all but finitely many $X_{i}$ 's, say $X_{p 1}, X_{p 2}, \ldots, X_{p n}$. For each $i \in\{1,2, \ldots, n\}, U(1 / P i, 0)$ contains all but finitely many points on $X_{p i}$. The space $X$ is not $C$-s-compact. To prove this, consider the closed set $A=\{(1 / n, 0): n \in \mathbb{N}\} \cup\{(0,0)\}$. Consider the semiopen cover

$$
\begin{gathered}
u=\left(\left\{\left(\frac{1}{2 n}, 0\right)\right\} \cup\left\{\left(\frac{1}{2 n}, \frac{1}{m}\right): n, m \in \mathbb{N}\right\} \cup\{(0,0)\}\right) \\
\cup\left(\{ ( \frac { 1 } { ( 2 n - 1 ) } , 0 ) \} \cup \left\{\left(\frac{1}{(2 n-1)}, \frac{1}{m}\right): m>j\right.\right. \\
\text { for some } j \in \mathbb{N}: n, m \in \mathbb{N}\}) .
\end{gathered}
$$

There is no finite $\mathscr{S} \subseteq \mathcal{U}$ such that $A \subseteq \bigcup_{\mathscr{S}} \mathrm{cl} U$. Hence, $X$ is not $C$-s-compact. 
An example of an $S$-closed space which is not $C$-s-compact is given as follows.

EXAMPLE 4.3. Let $D$ be the set of positive integers with discrete topology. Then the Katětov expansion $k D$ is extremally disconnected and $H$-closed and hence is $S$-closed. But it is not compact and hence not $C$-s-compact.

In a class of Hausdorff spaces, the following implication diagram is valid, no implication is reversible, in general, other than the following one:

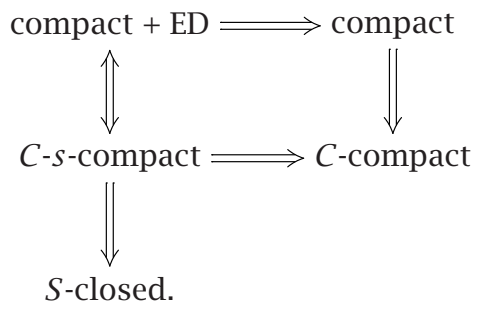

The following lemma is a corollary to [13, Theorem 7].

LEMMA 4.4. For a compact Hausdorff space, the following are equivalent:

(i) $X$ is extremally disconnected;

(ii) $X$ is projective;

(iii) $X$ is $S$-closed.

The product of two $C$-s-compact spaces need not be $C$-s-compact as can be seen from the following example.

EXAMPLE 4.5. The Stone-Cech compactification $\beta \mathbb{N}$ of the set of natural numbers is compact, Hausdorff, and extremally disconnected and hence is $C$-s-compact. But $\beta \mathbb{N} \times \beta \mathbb{N}$ is compact, Hausdorff, and not extremally disconnected, and hence it is not $S$-closed in view of the above lemma. Therefore, it is not $C$-s-compact.

REMARK 4.6. In fact, if $D$ is an infinite discrete space, the Stone-Cech compactification $\beta D$ is compact, Hausdorff, and extremally disconnected. But $\beta D \times \beta D$ is not extremally disconnected, but is compact and Hausdorff.

ACKNOWLEDGMENT. This work was partially supported by Housing and Urban Development HUD Special Projects Grant B 98 SP MD 0074.

\section{REFERENCES}

[1] J. P. Clay and J. E. Joseph, A characterization of C-compact spaces, Proc. Amer. Math. Soc. 82 (1981), no. 4, 657-658.

[2] R. F. Dickman Jr. and R. L. Krystock, S-sets and S-perfect mappings, Proc. Amer. Math. Soc. 80 (1980), no. 4, 687-692.

[3] R. F. Dickman Jr. and A. Zame, Functionally compact spaces, Pacific J. Math. 31 (1969), 303-311.

[4] G. Goss and G. Viglino, Some topological properties weaker than compactness, Pacific J. Math. 35 (1970), 635-638.

[5] L. L. Herrington and P. E. Long, Characterizations of C-compact spaces, Proc. Amer. Math. Soc. 52 (1975), 417-426. 
[6] J. E. Joseph, Multifunctions and graphs, Pacific J. Math. 79 (1978), no. 2, 509-529.

[7] J. E. Joseph and M. H. Kwack, On S-closed spaces, Proc. Amer. Math. Soc. 80 (1980), no. 2, 341-348.

[8] J. E. Joseph, M. H. Kwack, and B. M. P. Nayar, Sequentially functionally compact and sequentially C-compact spaces, Scientiae Mathematicae 2 (1999), no. 2, 187-194.

[9] N. Levine, Semi-open sets and semi-continuity in topological spaces, Amer. Math. Monthly 70 (1963), 36-41.

[10] B. M. P. Nayar, A remark on C-compact spaces, J. Austral. Math. Soc. Ser. A 64 (1998), no. 3, 327-328.

[11] T. Noiri, A generalization of closed mappings, Atti Accad. Naz. Lincei Rend. Cl. Sci. Fis. Mat. Natur. (8) 54 (1973), 412-415.

[12] S. Sakai, A note on C-compact spaces, Proc. Japan Acad. 46 (1970), 917-920.

[13] T. Thompson, $S$-closed spaces, Proc. Amer. Math. Soc. 60 (1976), 335-338.

[14] N. V. Veličko, $H$-closed topological spaces, Mat. Sb. (N.S.) 70 (1966), 98-112 (Russian), translated in Amer. Math. Soc. Transl. 78 (1968), 103-118.

[15] G. Viglino, C-compact spaces, Duke Math. J. 36 (1969), 761-764.

Bhamini M. P. Nayar: Department of Mathematics, Morgan State University, Baltimore, MD 21251, USA

E-mail address: bnayar@morgan.edu 


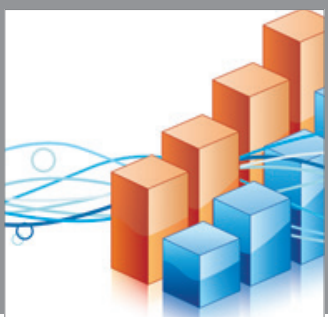

Advances in

Operations Research

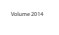

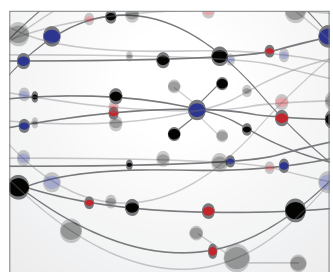

\section{The Scientific} World Journal
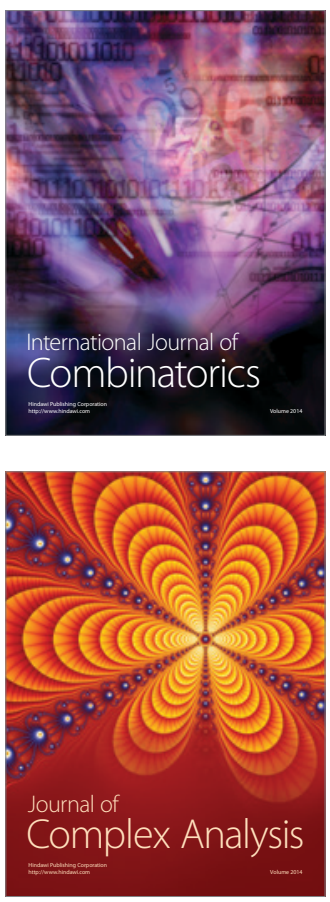

International Journal of

Mathematics and

Mathematical

Sciences
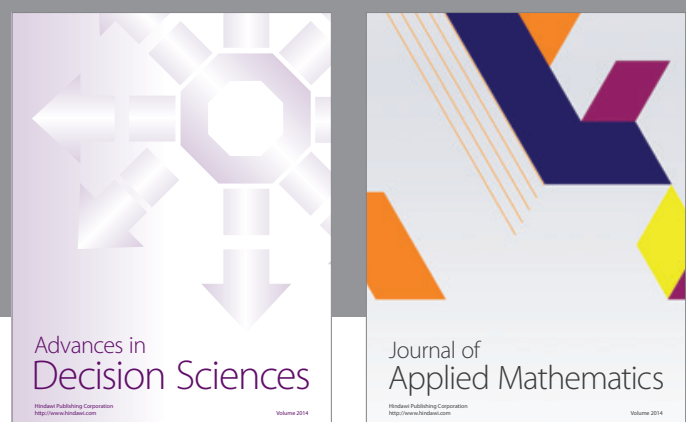

Journal of

Applied Mathematics
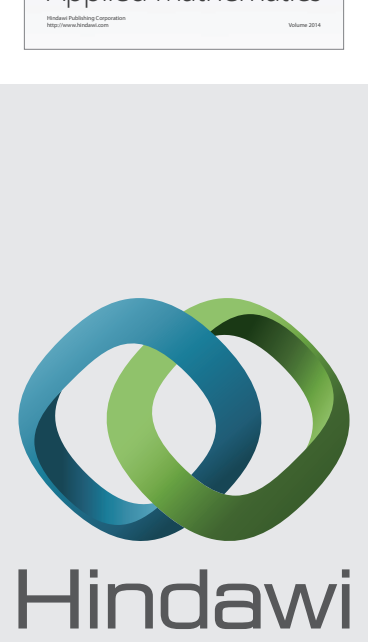

Submit your manuscripts at http://www.hindawi.com
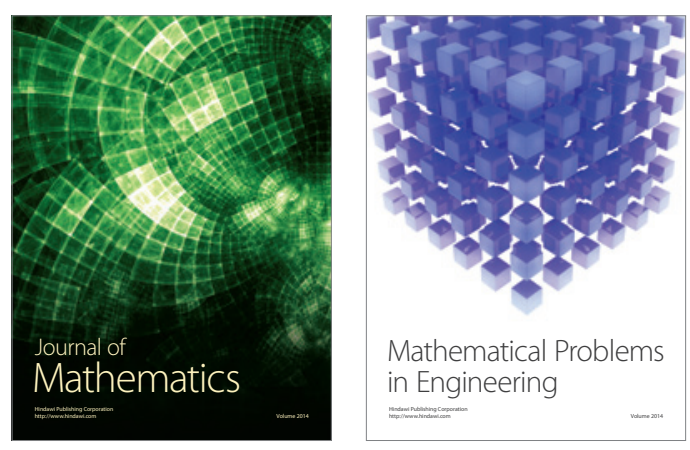

Mathematical Problems in Engineering
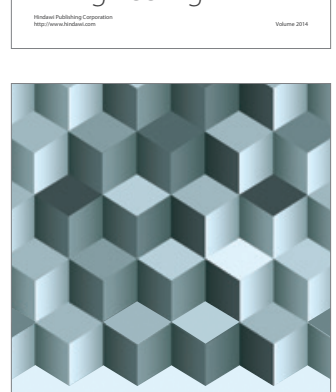

Journal of

Function Spaces
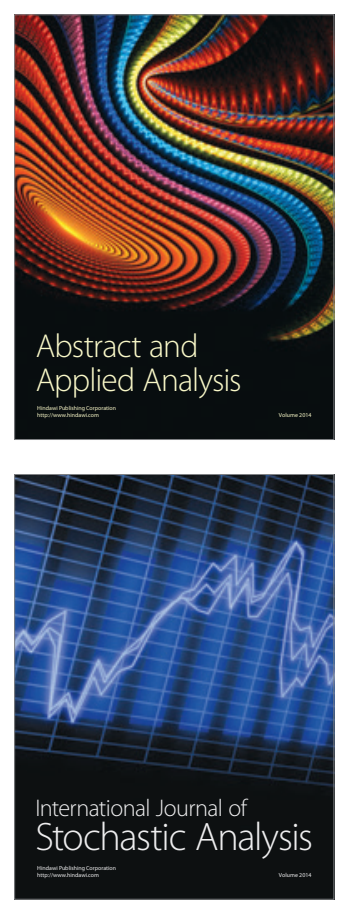

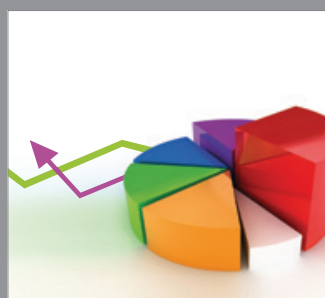

ournal of

Probability and Statistics

Promensencen
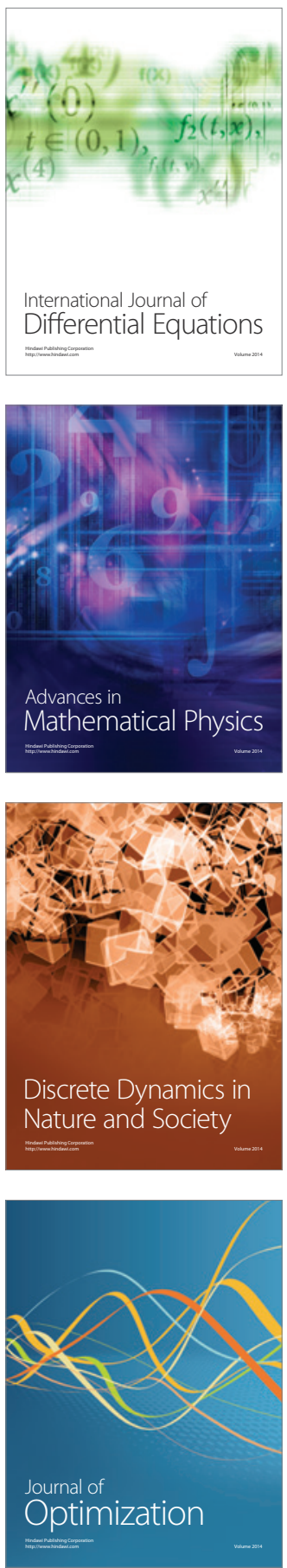